\title{
Chiral separation of underivatized and dansyl amino acids by ligand-exchange micellar electrokinetic capillary chromatography using a copper(II)-L-valine complex as selector
}

\author{
Zhi-Xia Zheng, Jin-Ming Lin*, Feng Qu \\ Research Center for Eco-Environmental Sciences, Chinese Academy of Sciences, P.O. Box 2871, Beijing 100085, China
}

Received 9 January 2003; received in revised form 20 May 2003; accepted 21 May 2003

\begin{abstract}
Ligand-exchange micellar electrokinetic capillary chromatography was used for the chiral resolution of underivatized and dansyl amino acid enantiomers simultaneously. The separation was achieved by chiral copper(II)-L-valine complexes incorporated in micelles of sodium dodecyl sulfate (SDS). The enantioresolution was strongly affected by SDS and a concentration of $20 \mathrm{~m} M$ SDS was shown to be necessary for the separation. Other impacting factors were investigated including $\mathrm{pH}$, the molar ratio of copper(II) to L-valine and the total concentration of complex. Using the proposed method, 11 different dansyl amino acids and two underivatized amino acids were separated successfully with a running electrolyte of $20 \mathrm{mM} \mathrm{NH} \mathrm{NH}_{4} \mathrm{OAc}, 4 \mathrm{mM} \mathrm{CuSO}_{4}, 8 \mathrm{~m} M$ L-valine and $20 \mathrm{~m} M$ SDS at pH 9.0 in less than $25 \mathrm{~min}$. Experiments were also performed with other amino acid ligands in order to vary the stability and the sterical arrangement of the copper(II) complexes and the possible chiral recognition mechanism was also discussed briefly.
\end{abstract}

(c) 2003 Elsevier B.V. All rights reserved.

Keywords: Ligand-exchange micellar electrokinetic capillary chromatography; Enantiomer separation; Amino acids; Amino acids, Dns derivatives; Valine

\section{Introduction}

Amino acids are essential components of many natural molecules and frequently found in food, feeds, body fluids and tissues. Chiral analysis of DL-amino acids is of great interest and of increasing importance in life science and many other related fields. Enantioseparation of racemic amino acids has been extensively studied by gas chromatography (GC) [1] and high-performance liquid chromatog-

*Corresponding author. Tel./fax: +86-10-6284-1953.

E-mail address: jmlin@mail.rcees.ac.cn (J.-M. Lin). raphy (HPLC) [2] using chiral stationary phases or chiral mobile phase additives. In recent years, capillary electrophoresis (CE) has been receiving extensive attention for chiral analysis because of its high separation efficiency, short analysis times, feasibility in selectivity manipulation, low cost and relative simple instrument setup [3-5], compared with other chromatographic techniques. On the basis of the ligand-exchange (LE) mechanism introduced by Davankov and Rogozhin in the early 1970s for HPLC [6], chiral resolution of dansyl amino acids by $\mathrm{CE}$ was firstly reported by Zare and co-workers using L-histidine- [7] or aspartame-Cu(II) complexes [8] as chiral selectors. Subsequently, a great 
variety of papers dealing with LE-CE enantioseparation of amino acids have been published using $\mathrm{Cu}(\mathrm{II})$-amino acid complexes as chiral selectors [914]. The authors observed a significant improvement in resolution when a micelle forming surfactant such as sodium dodecyl sulfate (SDS) was added to the electrolyte performing micellar electrokinetic chromatography (MEKC). Cohen et al. [15] and later, Sundin et al. [16] described the use of a complex of copper(II) and $N, N$-didecylalanine as chiral additive in combination with $20 \mathrm{~m} M$ SDS to facilitate separation of dansylated amino acids. Recently, Chen et al. [17,18] carefully studied chiral separation behaviors of the positional isomers and enantiomers of fluorophenylalanine and tyrosine with and without SDS addition. The authors called the method ligandexchange micellar electrokinetic capillary chromatography. As a hybrid mode of possessing both the advantage of high enantioselectivity in ligand-exchange mechanism and the main advantages of MEKC, LE-MEKC allows the manipulation of the selectivity for large classes of neutral and charged compounds, making possible separation that otherwise are not feasible by using only the mode of LE or MEKC. Using this combined separation mode, hydroxy acids and dipeptides, as well as 16 positional and optical isomers of tryptophan derivatives could be optically resolved [19]. The same group also proposed a method for the determination of the critical micelle concentration (CMC) of anionic surfactants based on the LE-MEKC principle [5].

Separation of underivatized amino acid enantiomers by $\mathrm{CE}$ has always been a challenge. Although reports on $\mathrm{CE}$ separations of tagged amino acid enantiomers are numerous, only a few chiral CE procedures have been reported for native amino acids [20]. In the present work, we describe an optical resolution system for the simultaneous enantiomeric separation of native and dansyl amino acids by LE-MEKC. Using copper(II) complexes of L-valine as chiral selectors, the resolution of 11 dansyl amino acids and two unmodified amino acids is achieved. The mechanism of chiral discrimination is discussed on the base of the stabilities of the copper(II) binary complexes and of their ternary diasteremeric complexes with amino acids formed in solution and SDS micelles.

\section{Experimental}

\subsection{Chemicals}

All dansyl (Dns) DL-amino acids including aspartic acid (Asp), glutamic acid (Glu), leucine (Leu), methionine (Met), $\alpha$-amino- $n$-butyric acid (Nbu), norleucine (Nle), norvaline (Nva), phenylalanine (Phe), threonine (Thr), tryptophan (Trp) and valine (Val) were purchased from Sigma (St. Louis, MO, USA). Underivatized DL-Phe was from Tokyo Kasei Kogyo (Tokyo, Japan); DL-Trp was from Nacalai Tesque (Kyoto, Japan). L-Val, L-Glu, L-alanine (Ala), L-serine (Ser) and L-ornithine (Orn) were of biochemical-reagent grade from the Institute of Shanghai Chemical Reagents Factory (Shanghai, China). Copper(II) sulfate pentahydrate $\left(\mathrm{CuSO}_{4} \cdot 5 \mathrm{H}_{2} \mathrm{O}\right)$ was from Kanto (Tokyo, Japan); SDS was obtained from Acros Organics (NJ, USA). Other chemicals were all of analytical-reagent grade obtained from Beijing Chemical Factory (China) and used as received. Standard solutions of amino acids were obtained by dissolving amino acids in $20 \mathrm{~m} M$ ammonium acetate $\left(\mathrm{NH}_{4} \mathrm{OAc}\right)$ solution and the concentrations of all stock solutions were $1.0 \mathrm{mM}$. Sample solutions were prepared by dilution of stock solutions in $20 \mathrm{mM}$ $\mathrm{NH}_{4} \mathrm{OAc}$ at the concentration range of $10^{-4} M$. The running electrolyte used for the enantiomeric separation, unless otherwise noted, consisted of $4 \mathrm{mM}$ $\mathrm{CuSO}_{4}, 8 \mathrm{~m} M \mathrm{~L}-\mathrm{Val}$ and $20 \mathrm{~m} M$ SDS in $20 \mathrm{~m} M$ $\mathrm{NH}_{4} \mathrm{OAc}$ adjusted to a desired $\mathrm{pH}$ by addition of $1 M$ aqueous ammonia or $1 M$ acetic acid. Water used to prepare sample and buffer solutions was freshly deionized by EASYpure water purification system with a $0.2-\mu \mathrm{m}$ fiber filter (Barnstead, USA).

\subsection{Instrumentation}

All experiments were performed with a Beckman P/ACE MDQ CE system (Fullerton, CA, USA) equipped with a photodiode array detector. Sample detection was performed at $214 \mathrm{~nm}$. Electropherograms were recorded and integrated by an IBM personal computer with Beckman 32 Karat software. Uncoated fused-silica capillaries (GL Sciences, Tokyo, Japan) with an effective length of $50 \mathrm{~cm}$ (57 $\mathrm{cm} \times 50 \mu \mathrm{m}$ I.D. $\times 375 \mu \mathrm{m}$ O.D.) were used for 
separation. The capillary temperature was maintained at $25^{\circ} \mathrm{C}$ by the cooling system of the CE instrument. Samples were injected hydrodynamically at 0.5 p.s.i. for $5 \mathrm{~s}$ and separated at $20 \mathrm{kV}(1$ p.s.i. $=6894.76 \mathrm{~Pa})$. To clean and activate the inner surface, new capillaries were flushed successively for $5 \mathrm{~min}$ with methanol, 5 min with $0.1 \mathrm{M} \mathrm{HCl}, 10$ min with $0.1 \mathrm{M}$ $\mathrm{NaOH}$, and 5 min with water. Daily before use, the capillary was rinsed for $2 \mathrm{~min}$ with $0.1 \mathrm{mM} \mathrm{NaOH}, 2$ min with water and 4 min with running electrolyte. Between analyses, the capillaries were rinsed for 4 min with running electrolyte.

\section{Results and discussion}

Val proved to be a good chiral selector for HPLC [21]. However, until now, there has been no report in CE. Since the use of HPLC needs large consumption of chiral eluent, development and application of an $\mathrm{L}-\mathrm{Val}-\mathrm{Cu}(\mathrm{II})$ complex as a chiral selector in $\mathrm{CE}$ will supply a powerful alternative to HPLC. When such a copper(II) complex was used as chiral additive to the running electrolyte for capillary zone electrophoretic analysis, resolution was not achieved for the racemic compounds studied (Fig. 1a), which suggests that the difference in stabilities of the ternary complexes is not enough for the separation. Therefore, the LEMEKC mode was chosen, a hybrid method of electrophoresis and chromatography with SDS as a micelle-forming agent for increasing the separation selectivity (Fig. 1b).

\subsection{Effect of SDS concentration}

The influence of the concentration of SDS was studied in the range from 0 to $40 \mathrm{~m} M$ using the running electrolyte containing $20 \mathrm{mM} \mathrm{NH}_{4} \mathrm{OAc}$, $4 \mathrm{mM} \mathrm{Cu}(\mathrm{II})$ and $8 \mathrm{~m} M$ L-Val at $\mathrm{pH}$ 9.0. In this work, Dns-Nbu, Dns-Met, Dns-Leu and Dns-Nle were used as model compounds to study the effects on separation. The results are shown in Fig. 2. As expected, addition of SDS resulted in a significant improvement in resolution. When SDS concentration was lower than $8.1 \mathrm{mM}$, the separation could not be carried out well. The separation gradually became better when concentration of SDS increased to $10 \mathrm{~m} M$ and the resolution increased up to a con-



Fig. 1. Electropherograms of the separation of the mixture of Dns-Nbu, Dns-Met, Dns-Leu and Dns-Nle using L-Val as a chiral ligand without (a) or with (b) $20 \mathrm{~m} M$ SDS. Conditions: $4 \mathrm{mM}$ CuSO$_{4}, 8 \mathrm{~m} M$ L-Val, $20 \mathrm{~m} M \mathrm{NH}_{4} \mathrm{OAc}$ at pH 9.0; injection, 0.5 p.s.i., 5 s; separation voltage, $20 \mathrm{kV}(350 \mathrm{~V} / \mathrm{cm})$; capillary, $57 \mathrm{~cm} \times 50 \mu \mathrm{m}$ I.D; capillary temperature, $25^{\circ} \mathrm{C}$. 




Fig. 2. Influence of SDS concentration on chiral separation in the presence of $4 \mathrm{mM} \mathrm{CuSO}_{4}, 8 \mathrm{mM}$ L-Val, $20 \mathrm{mM} \mathrm{NH}_{4} \mathrm{OAc}$ at $\mathrm{pH}$ 9.0; other conditions as in Fig. 1.

centration of $20 \mathrm{~m} M$. Above $20 \mathrm{~m} M$ SDS, the $R_{s}$ values slightly decreased for Dns-Leu and Dns-Nle while leveled off for Dns-Nbu and Dns-Met. A too high concentration of SDS resulted in a marked increase in separation time and the running current. Therefore, $20 \mathrm{~m} M$ SDS was selected as the optimum condition.

Addition of SDS micelles plays a crucial role in separation. This is due to an additional separation mechanism described as micellar electrokinetic chromatography introduced by Terabe et al. [22]. The formation of SDS micelles in the electrolyte solution was regarded as hydrophobic pseudo-stationary phase and the enantiomers with different stereoconformation and hydrophobicity will partition between aqueous buffer and micellar phase [16] associated with the chiral selector of the $\mathrm{Cu}$ (II)-Val complex with different partition coefficients. Meanwhile, enantiomers partitioned within micelles will undergo ligand exchange with one of the ligands of the complex [17]. Thus, a synergic effect of differences in the partition coefficient and complex formation constant may be generated between the chiral complex and SDS to enhance the resolution.

\subsection{Effect of $p H$}

An important factor in LE-MEKC is the $\mathrm{pH}$ of the running electrolyte, since complex formation is strongly dependent on $\mathrm{pH}$ because of the different dissociation degree of both the amino acids and the chiral selector ligand. The effect of the $\mathrm{pH}$ value of the running electrolyte was investigated in this work. The dependence of resolution of DL-amino acids on the $\mathrm{pH}$ value of the running electrolyte was studied over the $\mathrm{pH}$ range of 6.0 9.5 using $20 \mathrm{mM} \mathrm{NH}_{4} \mathrm{OAc}$ buffer consisting of $20 \mathrm{~m} M$ SDS, $4 \mathrm{mM} \mathrm{Cu}{ }^{2+}$ and $8 \mathrm{~m} M$ L-Val. The $\mathrm{pH}$ value of buffer was adjusted by adding $1 M \mathrm{NH}_{3} \cdot \mathrm{H}_{2} \mathrm{O}$ or acetic acid (HAc). The $\mathrm{pH}$ effects were not studied over $\mathrm{pH} 9.5$ in order to avoid the precipitation of $\mathrm{Cu}(\mathrm{OH})_{2}$. As shown in Fig. 3, the separation of Dns-amino acids increased apparently with the increasing of $\mathrm{pH}$ range from 6.0 to 8.5 , then decreased at the $\mathrm{pH}$ value above 8.5 . The distinct maximum of the chiral separation could be achieved at $\mathrm{pH} 8.5$. As $\mathrm{pH}$ increases from 6.0 to 8.5, the free $\mathrm{L}-\mathrm{Val}$ is changed from the neutral form to negative ions [at its isoelectric point $(\mathrm{p} I)$ around 6.0]. As a result, the stability of the formed $\mathrm{Cu}(\mathrm{II})-$ L-Val complex will be increased. Thus, the chiral resolution should be enhanced. But further increasing of $\mathrm{pH}$ will lead to too high stability of the $\mathrm{Cu}$ (II)ligand complex, which makes it difficult for the analyte enantiomers to replace ligands in the $\mathrm{Cu}$ (II)ligand complex. These effects result in a maximum $\mathrm{pH}$ of 8.5 for the chiral separation. However, the



Fig. 3. Influence of $\mathrm{pH}$ on the resolution of Dns-amino acids. Buffer: $20 \mathrm{mM} \mathrm{NH} \mathrm{NH}_{4} \mathrm{OAc}$ containing $4 \mathrm{mM} \mathrm{CuSO}_{4}, 8 \mathrm{mM}$ L-Val and $20 \mathrm{~m} M$ SDS. 
migration time decreased markedly at higher $\mathrm{pH}$ value resulting from the increase of electroosmotic mobility. Taking into consideration of resolution and migration time simultaneously, running buffer at $\mathrm{pH}$ 9.0 was selected to yield better resolution and shorter separation time.

\subsection{Effect of the composition of the $\mathrm{Cu}(\mathrm{II})-\mathrm{L}-\mathrm{Val}$ complex}

Achiral separation by MEKC was performed but the Dns-amino acids could not be resolved. When the $\mathrm{Cu}(\mathrm{II})-\mathrm{L}-\mathrm{Val}$ complex was present in the running electrolyte, the poor separation was greatly improved. To determine the optimum selector concentration, the $\mathrm{Cu}(\mathrm{II})-\mathrm{L}-\mathrm{Val}$ complex concentration was increased from 2 to $10 \mathrm{~m} M$ at an increment of $2 \mathrm{~m} M$ while the molar ratio between copper and the chelating amino acid was kept always at 1:2. The results reported in Table 1 show a general decrease of the migration time of the analyzed compounds by increasing the concentration of the $\mathrm{Cu}$ (II) complex. The resolution for the tested amino acids increased up to $4 \mathrm{mMCu}(\mathrm{II})-\mathrm{L}-$ Val complex and then decreased at higher concentration. The results are different from those reported in LE-MEKC [23] using chiral complex as buffer additive, in which the resolution always increased steadily with the concentration of the copper(II) complex. In our experiment, a $4 \mathrm{~m} M$ selector concentration allowed the best resolution for the amino acids investigated.

The molar ratio of $\mathrm{Cu}(\mathrm{II})$ to $\mathrm{L}-\mathrm{Val}$ has a strong influence on resolution. In order to make clear the effect of the molar ratio of metal ion to ligand on the

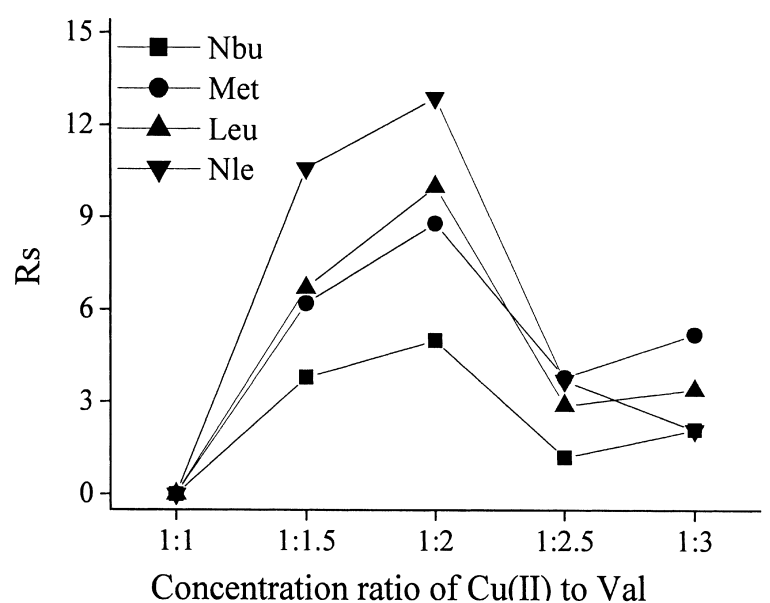

Fig. 4. Influence of the molar ratio of $\mathrm{Cu}^{2+}$ to $\mathrm{L}-\mathrm{Val}$ on resolution. Buffer: $20 \mathrm{~m} M$ ammonium acetate, $\mathrm{pH}$ 9.0, $20 \mathrm{~m} M$ SDS, the total concentration of $\mathrm{Cu}^{2+}$ and $\mathrm{L}-\mathrm{Val}$ is $12 \mathrm{mM}$.

enantiomeric separation, the relative amounts of the two components were varied between $1: 1$ to $1: 3$ $\left(\left[\mathrm{Cu}^{2+}\right]\right.$ :[Val], $\left.\mathrm{m} M / \mathrm{m} M\right)$, while the total concentration of $\mathrm{Cu}(\mathrm{II})$ and $\mathrm{L}$-Val was kept constant at $12 \mathrm{mM}$. Fig. 4 indicates that the resolution of Dnsamino acids was very sensitive to the ratio and the separation increased rapidly as the ratio increased from 1:1 to 1:2. A large excess of L-Val dramatically reduced the resolutions, which may be due to the reduction in the competitive opportunity for the solutes to form complex with $\mathrm{Cu}^{2+}$. Therefore, the optimum separation was obtained at a ratio of $1: 2$ $\left(\left[\mathrm{Cu}^{2+}\right]\right.$ :[Val] $)$, indicating that $\mathrm{L}-\mathrm{Val}$ forms a biscomplex with $\mathrm{Cu}(\mathrm{II})$ ion.

Table 1

Influence of concentration $(C)$ of $\mathrm{Cu}(\mathrm{II})$ and $\mathrm{L}-\mathrm{Val}$ on separation by $\mathrm{MEKC}$

\begin{tabular}{|c|c|c|c|c|c|c|c|c|c|c|c|c|}
\hline \multirow{2}{*}{$\begin{array}{l}C \\
(\mathrm{~m} M: \mathrm{m} M)\end{array}$} & \multicolumn{3}{|c|}{ Dns-Nbu } & \multicolumn{3}{|c|}{ Dns-Met } & \multicolumn{3}{|c|}{ Dns-Leu } & \multicolumn{3}{|c|}{ Dns-Nle } \\
\hline & $\begin{array}{l}t_{\mathrm{L}} \\
(\min )\end{array}$ & $\begin{array}{l}t_{\mathrm{D}} \\
(\min )\end{array}$ & $R_{s}$ & $\begin{array}{l}t_{\mathrm{L}} \\
(\min )\end{array}$ & $\begin{array}{l}t_{\mathrm{D}} \\
(\mathrm{min})\end{array}$ & $R_{s}$ & $\begin{array}{l}t_{\mathrm{L}} \\
(\min )\end{array}$ & $\begin{array}{l}t_{\mathrm{D}} \\
(\min )\end{array}$ & $R_{s}$ & $\begin{array}{l}t_{\mathrm{L}} \\
(\min )\end{array}$ & $\begin{array}{l}t_{\mathrm{D}} \\
(\min )\end{array}$ & $R_{s}$ \\
\hline 0 & 5.495 & 5.495 & 0 & 5.747 & 5.747 & 0 & 6.587 & 6.587 & 0 & 6.920 & 6.920 & 0 \\
\hline $2: 4$ & 9.517 & 9.754 & 1.8 & 10.463 & 11.113 & 3.8 & 12.637 & 13.283 & 3.4 & 15.096 & 17.437 & 7.9 \\
\hline $4: 8$ & 8.617 & 9.459 & 5.0 & 9.713 & 10.679 & 8.8 & 12.201 & 12.530 & 10.0 & 13.721 & 15.483 & 12.9 \\
\hline $6: 12$ & 8.188 & 8.492 & 3.4 & 8.767 & 9.750 & 6.1 & 9.946 & 11.736 & 5.9 & 11.924 & 14.570 & 9.4 \\
\hline $8: 16$ & 7.717 & 8.021 & 3.0 & 8.333 & 9.024 & 5.8 & 9.132 & 9.941 & 5.8 & 10.138 & 11.909 & 8.8 \\
\hline $10: 20$ & 7.450 & 7.563 & 1.2 & 7.858 & 8.162 & 3.0 & 8.371 & 8.625 & 2.1 & 8.988 & 9.588 & 3.3 \\
\hline
\end{tabular}

Running electrolyte: $20 \mathrm{mM} \mathrm{NH} \mathrm{NH}_{4} \mathrm{OAc}, 20 \mathrm{mM}$ SDS and $\mathrm{Cu}(\mathrm{II})-\mathrm{L}-\mathrm{Val}(1: 2)$; $\mathrm{pH} 9.0$, other conditions as in Fig. $1 . t_{\mathrm{L}}$ and $t_{\mathrm{D}}$ are the migration times of the $\mathrm{L}-$ and $\mathrm{D}$-enantiomers, respectively. 


\subsection{Enantioseparation of dansyl- and underivatized-DL-amino acids}

The recommended experimental conditions were as follows: $20 \mathrm{~m} M$ SDS, $4 \mathrm{~m} M \mathrm{CuSO}_{4}$ and $8 \mathrm{~m} M$ L-Val, $20 \mathrm{mM} \mathrm{NH} 4 \mathrm{OAc}, \mathrm{pH}$ 9.0. Fig. 5 shows the electropherogram of a mixture of underivatized Trp and seven different Dns-amino acid enantiomers. The results indicate that the L-enantiomers invariably migrated faster than the D-enantiomers. Under the proposed conditions, 11 pairs of different Dns-amino acids and two underivatized amino acids enantiomers could be successfully resolved and the migration time, separation factor and resolution are summarized in Table 2. As can be seen, the elution order of these amino acid derivatives simply agrees with the hydrophobic nature of side chains. In LE-MEKC, the elution order of enantiomers is basically dependent on both the stability of the ternary complexes and the partition coefficient of the enantiomers associated with the chiral selector of $\mathrm{Cu}(\mathrm{II})-\mathrm{L}$-Val complexes between the SDS micellar phase and the bulk electrolyte phase. As we have noted, the differences in stability between the ternary complexes formed by each of the enantiomers is difficult to determine in the $\mathrm{Cu}(\mathrm{II})-\mathrm{L}-\mathrm{Val}$ solution. And the migration order in
Table 2

Separation data for dansyl amino acids

\begin{tabular}{lcclr}
\hline Amino acid & $t_{\mathrm{L}}(\mathrm{min})$ & $t_{\mathrm{D}}(\min )$ & $\alpha$ & $R_{s}$ \\
\hline Dns-Val & 8.456 & 8.733 & 1.02 & 1.9 \\
Dns-Thr & 8.904 & 9.058 & 1.02 & 1.9 \\
Dns-Nva & 9.113 & 9.600 & 1.05 & 4.4 \\
Dns-Nbu & 9.537 & 9.908 & 1.04 & 4.9 \\
Dns-Asp & 10.050 & 10.262 & 1.02 & 1.5 \\
Dns-Met & 10.675 & 11.625 & 1.09 & 8.9 \\
Dns-Leu & 11.804 & 12.458 & 1.06 & 9.6 \\
Dns-Glu & 12.748 & 12.833 & 1.01 & 0.9 \\
Dns-Nle & 13.329 & 15.642 & 1.17 & 12.9 \\
Dns-Phe & 15.242 & 17.967 & 1.18 & 13.1 \\
Dns-Trp & 23.721 & 24.667 & 1.04 & 3.6 \\
Phe & 10.975 & 11.375 & 1.04 & 1.1 \\
Trp & 16.529 & 18.063 & 1.09 & 4.3 \\
\hline
\end{tabular}

Migration time, separation factor $(\alpha)$ and resolution $\left(R_{s}\right)$ are given.

Conditions: $20 \mathrm{~m} M \mathrm{NH}_{4} \mathrm{OAc}, 8 \mathrm{~m} M$ L-Val, $4 \mathrm{~m} M \mathrm{CuSO}_{4}, 20 \mathrm{~m} M$ SDS, adjusted with $\mathrm{NH}_{3} \cdot \mathrm{H}_{2} \mathrm{O}$ to $\mathrm{pH} 9.0$.

the present system is thought to be dependent on the partition coefficient. From Table 2, the Dns-Trp derivative with the most hydrophobic side chain partitioned between the micelle and the buffer with the greatest distribution percentage in the micelle showed the highest retention in the micellar phase and eluted lastly. These results suggest that the major



Fig. 5. Electropherogram of the optical resolution of underivatized and Dns-amino acid enantiomers. Running electrolyte contains $20 \mathrm{~m} M$ SDS, $20 \mathrm{mM} \mathrm{NH} \mathrm{NH}_{4} \mathrm{OAc}, 4 \mathrm{~m} M \mathrm{Cu}^{2+}$ and $8 \mathrm{~m} M$ L-Val, pH was adjusted at 9.0. 
factor involved in the recognition mechanism is the affinity of the complexes for the micellar phase, therefore, the distribution of an analyte between the micellar and aqueous phases plays the key role in the separation.

\subsection{Comparison of other amino acid ligands with L-Val}

An important component of the recognition process is the nature of the chiral ligand. In order to investigate whether chiral discrimination with copper(II) complexes of other amino acid ligands may occur, some other ligands, i.e., L-Ala, L-Glu, L-Orn and L-Ser were explored for the enantiomeric separation of Dns-amino acids under the same experimental condition and chiral separation ability was compared with that of L-Val. When L-Ala or L-Glu was used as chiral ligand, no chiral separation was achieved. L-Orn and L-Ser showed relative high resolution ability and the enantioseparation increases as a function of the hydrophobicity in the following order: $\mathrm{L}-\mathrm{Ser}<_{\mathrm{L}-O r n}<_{\mathrm{L}-V a l}$. The higher hydrophobic the ligand, the better its resolution ability. This might be due to its high hydrophobicity which allows additional binding between the hydrophobic side chain of the selector and SDS micelle interior. As stated above, the stereoselectivity in solution contributes but a minimum to the overall discrimination process in LE-MEKC and that the major factor involved in the recognition mechanism is the affinity of the analytes for micellar phase. These results are similar to the recognition mechanism reported for HPLC using complex as chiral additives in mobile phase [24,25]. It was assumed that two factors were responsible for chiral recognition in ligand-exchange chromatography according to the three-point interaction rule: bidentate coordination of two amino acids to a metal ion and additional interactions of a substituent of the amino acid with the support. Finally, it was stated that the difference in stability of the diastereomeric ternary complexes in aqueous phase has a negligible influence on enantioselectivity and the chiral recognition takes place on the column where the initial complex is adsorbed. Our results shows clearly that the approach of LE-MEKC can be considered as a type of ligand-exchange chromatography from the nature of the recognition mechanism.

\section{Conclusion}

An optical resolution system for enantioseparation using a $\mathrm{Cu}(\mathrm{II})-\mathrm{L}-\mathrm{Val}$ complex as chiral selector is developed. The chiral $\mathrm{Cu}$ (II)-L-Val complex shows effective enantioresolution for the study of dansyl amino acids and underivatized amino acid enantiomers in combination of SDS as achiral micelle forming regent. The procedure has been optimized in terms of SDS concentration, the running buffer $\mathrm{pH}$, the ternary complex composition and the concentration of the complex. Under optimum conditions, enantioseparation of 11 Dns-amino acids as well as two underivatized amino acids was achieved (see Table 2). This work will make an inspiration to develop the more useful CE chiral separation system and further efforts will be made to extend the applications of this method in real samples, such as some chiral drugs containing amino alcohol structures.

\section{Acknowledgements}

The authors would like to thank the financial support from the National Science Fund for Distinguished Young Scholars of China (No. 20125514), the National Nature Science Foundation of China (Nos. 50273046 and 20275043) and the National High Technology Research and Development Program of China (863 Program) (No. 2001AA630503).

\section{References}

[1] S.-C. Chang, R. Charles, E. Gil-Av, J. Chromatogr. 235 (1982) 87.

[2] T. Hobo, S. Suzuki, K. Watabe, E. Gil-Av, Anal. Chem. 57 (1985) 362.

[3] J.-M. Lin, T. Hobo, Chin. J. Anal. Sci. 16 (2000) 328.

[4] J.-M. Lin, Z. Chen, Acta Pharm. Sinica 34 (1999) 716.

[5] Z. Chen, J.-M. Lin, K. Uchiyama, T. Hobo, Anal. Chim. Acta 403 (2000) 173.

[6] V.A. Davankov, S.V. Rogozhin, J. Chromatogr. 60 (1971) 284.

[7] E. Gassmann, J.E. Kuo, R.N. Zare, Science 230 (1985) 813.

[8] P. Gozel, E. Gassmann, H. Michelsen, R.N. Zare, Anal. Chem. 59 (1987) 44. 
[9] A. Végvári, M.G. Schmid, F. Kilár, G. Gübitz, Electrophoresis 19 (1998) 2109.

[10] M.G. Schmid, R. Rinaldi, D. Dreveny, G. Gübitz, J. Chromatogr. A 846 (1999) 157.

[11] Z.-B. Yuan, L.-L. Yang, S.-S. Zhang, Electrophoresis 20 (1999) 1842.

[12] A. Karbaum, T. Jira, J. Chromatogr. A 874 (2000) 285.

[13] Z. Chen, M. Niitsuma, T. Nakagama, K. Uchiyama, T. Hobo, J. Sep. Sci. 25 (2002) 1197.

[14] Z. Chen, M. Niitsuma, K. Uchiyama, T. Hobo, J. Chromatogr. A 990 (2003) 75.

[15] A.S. Cohen, A. Paulus, B.L. Karger, Chromatographia 24 (1987) 15.

[16] N.G. Sundin, T.M. Dowling, N. Grinberg, G. Bicker, J. Microcol. Sep. 8 (1996) 323.

[17] Z. Chen, J.-M. Lin, K. Uchiyama, T. Hobo, J. Chromatogr. A 813 (1998) 369.
[18] Z. Chen, J.-M. Lin, K. Uchiyama, T. Hobo, J. Microcol. Sep. 11 (1999) 534.

[19] Z. Chen, J.-M. Lin, K. Uchiyama, T. Hobo, Chromatographia 49 (1999) 436.

[20] M.G. Schmid, G. Gübitz, Enantiomer 1 (1996) 23.

[21] S.V. Galushko, I.P. Shishkina, I.I. Gerus, M.T. Kolycheva, J. Chromatogr. 600 (1992) 83.

[22] S. Terabe, K. Otsuka, K. Ichikawa, A. Tsuchiya, T. Ando, Anal. Chem. 56 (1984) 111.

[23] X.-N. Lu, Y. Chen, L. Guo, Y.-F. Yang, J. Chromatogr. A 945 (2002) 249.

[24] V.A. Davankov, A.A. Kurganov, A.S. Bochkov, Adv. Chromatogr. (N. Y.) 22 (1983) 71.

[25] V.A. Davankov, in: V.A. Davankov, J.D. Narratil, H.F. Walton (Eds.), Ligand Exchange Chromatography, CRC Press, Boca Raton, FL, 1988, p. 67. 